\title{
ARTICLE \\ A randomized, placebo-controlled trial of extended-release guanfacine in children with autism spectrum disorder and ADHD symptoms: an analysis of secondary outcome measures
}

\author{
Laura C. Politte ${ }^{1}$, Lawrence Scahill ${ }^{2}$, Janet Figueroa ${ }^{3}$, James T. McCracken ${ }^{4}$, Bryan King ${ }^{5}$ and Christopher J. McDougle ${ }^{6}$
}

In a prior report, we showed that extended-release guanfacine (GEXR) is safe and effective for children with autism spectrum disorder (ASD) accompanied by ADHD symptoms. Here, we examine the impact of GEXR on oppositional behavior, anxiety, repetitive behavior, and sleep disturbance. Sixty-two subjects with ASD (53 boys, 9 girls; ages 5-14 years) were randomly assigned to GEXR $(n=30)$ or placebo ( $n=32)$ for 8 weeks. Outcomes include the Home Situation Questionnaire-Modified for ASD (HSQ-ASD), Anxiety scale of the Child and Adolescent Symptom Inventory (CASI), Children's Yale-Brown Obsessive-Compulsive Scale-Modified for ASD (CYBOCS-ASD), and Children's Sleep Habits Questionnaire (CSHQ). A repeated measures linear mixed model was used to determine the effects of treatment group and time on HSQ scores. For other measures, change from baseline was evaluated with Analysis of Covariance (ANCOVA).

After 8 weeks of treatment, parent ratings of oppositional behavior on the HSQ declined by $44 \%$ (per item mean from 3.4 to 1.9 ) in the GEXR group compared to $12 \%$ (from 3.3 to 2.9 ) for placebo $(p=0.004)$. Repetitive behavior on the CYBOCS-ASD showed a significantly greater decline in GEXR-treated participants compared to placebo ( $24 \% \mathrm{vs.}<1 \%, p=0.01)$. No group differences were observed on CASI Anxiety or CSHQ ( $p=0.64$ and 0.75 , respectively). GEXR was effective in reducing oppositional behavior and, more modestly, repetitive behavior. GEXR was not superior to placebo for anxiety, though baseline anxiety ratings were low. GEXR did not significantly improve sleep habits. Future studies could focus on repetitive behavior or anxiety, symptoms with limited treatment options.

Neuropsychopharmacology (2018) 43:1772-1778; https://doi.org/10.1038/s41386-018-0039-3

\section{INTRODUCTION}

Autism spectrum disorder (ASD) is a heterogeneous condition characterized by impaired social communication, restricted interests and repetitive behaviors [1] with an estimated worldwide prevalence of $0.6-0.7 \%[2,3]$. The etiology of ASD is poorly understood and likely involves interplay of genetic vulnerabilities and environmental exposures, resulting in an array of developmental and behavioral phenotypes. Across the range of severity, ASD is often associated with lifelong burden for affected individuals and their families. No medications are currently approved by the Food and Drug Administration (FDA) to treat core symptoms of ASD. However, commonly associated problems such as tantrums, aggression, oppositional behavior, anxiety, hyperactivity, and impulsiveness are frequently the target of pharmacological intervention in youth with ASD [4]. Accumulated evidence supports the use of methylphenidate, atomoxetine, and guanfacine to treat ADHD symptoms in children with ASD [5-11], yet treatment options for oppositional behavior, anxiety, and repetitive behavior are limited.
Guanfacine is a selective alpha ${ }_{2 \mathrm{~A}}$ adrenergic receptor agonist that stimulates postsynaptic alpha ${ }_{2 A}$ receptors in the prefrontal cortex (PFC), potentiating noradrenergic transmission and strengthening the connectivity of PFC networks [12,13]. This mechanism of action likely mediates the therapeutic effects of guanfacine on working memory, attention, and behavioral control [13]. The extended release formulation (GEXR) is approved by the FDA for treatment of ADHD in children and adolescents aged 6-17-year-old.

Our group recently reported the results of an 8-week, randomized, placebo-controlled trial of GEXR in 62 children (mean age 8.5 years) with ASD accompanied by hyperactivity and impulsiveness [10]. In that study, 15 of 30 (50\%) GEXR-treated participants were rated 'much improved' or 'very much improved' on the Clinical Global Impressions Improvement Scale (CGI-I) compared to 3 of $32(9.4 \%)$ in the placebo group. Scores on the Aberrant Behavior Checklist (ABC)-Hyperactivity subscale, the primary outcome, declined by a mean of $43.6 \%$ in the GEXR group compared to $13.2 \%$ in the placebo group. GEXR use was safe and generally well-tolerated in this sample.

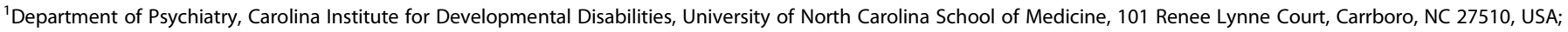

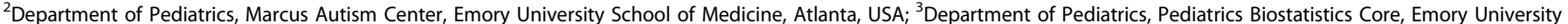

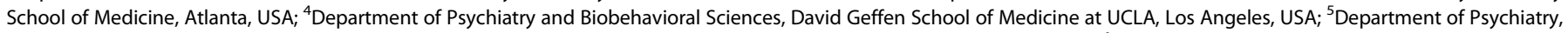

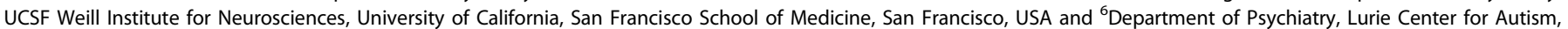
Harvard Medical School, Boston, USA

Correspondence: Laura C. Politte (laura.politte@cidd.unc.edu)
}

Received: 29 September 2017 Revised: 8 December 2017 Accepted: 14 February 2018 Published online: 27 February 2018 
Studies in non-ASD pediatric samples suggest that GEXR may be beneficial for oppositional behavior in ADHD and for treatment of anxiety disorders [14-16]. A pilot study by Murphy and colleagues showed no difference between GEXR and placebo for the treatment of tics [17]. The preliminary findings for oppositional behavior and anxiety are limited. Immediate-release alpha $_{2 \mathrm{~A}}$-agonists, including guanfacine and clonidine, are commonly prescribed "off label" in pediatric populations to treat insomnia due to their sedating properties [18]. However, a recent placebo-controlled study of GEXR in 29 children with ADHD and co-occurring sleep problems was terminated early after finding that GEXR was associated with decreased total sleep time on polysomnography compared with placebo [19].

In this analysis of secondary outcome measures from a randomized, placebo-controlled trial of GEXR in children with ASD and significant symptoms of ADHD [10], we tested whether GEXR would result in greater improvements in oppositional behavior, anxiety, repetitive behavior, and sleep disturbance than placebo.

\section{METHODS}

Study design

Details of the study design have been previously published [10]. Briefly, participants were randomly assigned 1:1 to GEXR or placebo at one of 5 sites (University of California at Los Angeles, Emory University, Massachusetts General Hospital, University of Washington at Seattle, and Yale University) for 8 weeks. Randomization was conducted within sites using permuted blocks. The primary outcome measure was the parent-rated, 16-item Aberrant Behavior Checklist-Hyperactivity subscale that includes overactivity, impulsiveness, inattention, and oppositional behavior in youth with developmental disabilities [20]. Screening assessments included routine laboratory tests (blood counts, electrolytes, glucose, liver function tests, blood urea nitrogen, creatinine, and urinalysis), medical and psychiatric histories, physical examination, vital signs, and routine electrocardiogram (ECG). Subjects were assessed by a treating clinician and an independent evaluator weekly for the first 4 weeks and then at weeks 6 and 8 during the 8 -week double-blind phase. Blood pressure, pulse, height, weight, and adverse events were monitored at every visit; an ECG was repeated at Week 8.

Written informed consent was obtained from a parent (or guardian) before study data were collected; capable children also provided assent. The study was approved by the local institutional review boards at each of the 5 sites and registered with Clinicaltrials.gov (NCT01238575). Data integrity was monitored by the coordinating centers (Yale University and Emory University), and an external monitoring board reviewed safety, enrollment, and attrition three times per year. Study procedures were conducted in accordance with the ethical standards of the Helsinki Declaration of 1975.

Participants

To be eligible, participants had to be between 5-year-old and 14-year-old and meet DSM-IV criteria for autistic disorder, Asperger's disorder, or pervasive developmental disorder not otherwise specified. The diagnoses followed a multidisciplinary team evaluation corroborated by the Autism Diagnostic Observation Schedule (ADOS) [21] and Social Communication Questionnaire (SCQ) [22]. Subjects were required to have an intelligence quotient (IQ) of at least 35 and moderate or greater levels of hyperactivity, impulsiveness, and distractibility, with a minimum entry score of 24 on the ABC-Hyperactivity subscale [20] and a Clinical Global Impressions-Severity (CGI-S) [23] score of moderate or greater. Anticonvulsant medication for treatment of seizures was allowed if the dose was stable for at least 4 weeks prior to study entry and the participant had been seizure-free for at least
6 months. Other than stable anticonvulsant treatment for seizures, participants had to be medication-free. Children with significant medical illness, another psychiatric illness or behavioral problems in need of immediate treatment, inability to swallow pills whole, and females with a positive urine pregnancy test were excluded.

Treatment

Using a fixed-flexible dosing regimen, children weighing $<25 \mathrm{~kg}$ received up to $3 \mathrm{mg} /$ day placebo or GEXR and children weighing $25 \mathrm{~kg}$ or more received up to $4 \mathrm{mg} /$ day, with no upward adjustments after day 28. The medication dose began with 1 $\mathrm{mg}$ /day with planned dose increases weekly for participants $\geq 25$ $\mathrm{kg}$. Children weighing $<25 \mathrm{~kg}$ stayed with $1 \mathrm{mg}$ for two weeks. The treating clinician could delay a planned increase or reduce the dose to manage a suspected adverse event. The dose could also be moved from morning to evening to manage excessive daytime drowsiness.

Table 1. Selected baseline characteristics of children with ASD randomly assigned to GEXR or placebo

\begin{tabular}{|c|c|c|c|c|c|c|}
\hline & \multicolumn{2}{|c|}{$\begin{array}{l}\text { Guanfacine } \\
(n=30)\end{array}$} & \multicolumn{2}{|c|}{$\begin{array}{l}\text { Placebo ( } n \\
=32)\end{array}$} & & \multirow[t]{2}{*}{$P$ value } \\
\hline & $N$ & $\%$ & $N$ & $\%$ & & \\
\hline Males & 26 & 86.7 & 27 & \multicolumn{2}{|c|}{84.4} & 0.99 \\
\hline \multicolumn{6}{|l|}{ DSM-IV diagnosis } & \multirow[t]{4}{*}{0.31} \\
\hline Autistic disorder & 25 & 83.3 & 26 & 81.2 & & \\
\hline PDD-NOS & 3 & 10.0 & 6 & 18.7 & & \\
\hline Asperger's disorder & 2 & 6.7 & 0 & 0 & & \\
\hline \multicolumn{7}{|l|}{ Intelligence score } \\
\hline $\mathrm{IQ}<70^{\mathrm{a}}$ & 11 & 36.7 & 10 & \multicolumn{2}{|c|}{33.0} & 0.79 \\
\hline$I Q \geq 70$ & 19 & 63.3 & 20 & \multicolumn{2}{|c|}{67.0} & \\
\hline \multicolumn{6}{|l|}{ CGI-Severity } & \multirow[t]{4}{*}{$0.63^{b}$} \\
\hline Moderately ill & 12 & 40.0 & 12 & 37.5 & & \\
\hline Markedly ill & 17 & 56.7 & 17 & 53.1 & & \\
\hline Severely ill & 1 & 3.3 & 3 & 9.4 & & \\
\hline \multicolumn{6}{|l|}{ Tanner stage } & \multirow[t]{3}{*}{0.99} \\
\hline Tanner 1 or 2 & 29 & 96.7 & 31 & \multicolumn{2}{|c|}{96.7} & \\
\hline \multirow[t]{2}{*}{ Tanner 3} & 1 & 3.3 & 1 & \multicolumn{2}{|l|}{3.1} & \\
\hline & & Mean & SD & Mean & SD & $P$-value \\
\hline $\begin{array}{l}\text { Social communication } \\
\text { questionnaire }\end{array}$ & & 23.9 & 6.4 & 21.4 & 6.4 & 0.15 \\
\hline \multicolumn{7}{|l|}{ VABS II } \\
\hline Socialization & & 66.8 & 14.3 & 67.1 & 14.9 & 0.93 \\
\hline Communication & & 72.9 & 20.1 & 73.5 & 13.7 & 0.40 \\
\hline Daily living & & 72.7 & 19.9 & 74.1 & 15.9 & 0.77 \\
\hline HSQ-ASD (per-item mean) & & 3.4 & 1.6 & 3.3 & 1.4 & 0.74 \\
\hline CYBOCS-ASD & & 11.2 & 5.1 & 10.9 & 5.4 & 0.79 \\
\hline CASI-Anxiety & & 14.0 & 10.2 & 15.8 & 11.1 & 0.62 \\
\hline $\mathrm{CSHQ}$ & & 44.0 & 4.9 & 44.4 & 44.0 & 0.67 \\
\hline \multicolumn{7}{|c|}{$\begin{array}{l}\text { aIQ missing in } 2 \text { subjects in placebo group } \\
\text { bThe } p \text { value represents the proportion of subjects }>\text { moderate on Clinical } \\
\text { Global Impression-Severity by group } \\
\text { VABS II Vineland adaptive behavior scales, 2nd edition } \\
\text { HSQ home situation questionnaire } \\
\text { CYBOCS-ASD children's Yale-Brown obsessive compulsive scale, modified } \\
\text { for ASD } \\
\text { CASI-Anxiety child and adolescent symptom inventory-anxiety subscale } \\
\text { CSHQ children's sleep habits questionnaire }\end{array}$} \\
\hline
\end{tabular}




\section{Outcome measures}

Home situations questionnaire-modified for autism spectrum disorder (HSQ-ASD). The original HSQ is a 16-item caregiver report designed to measure oppositional behavior in children with disruptive behavior disorders in a variety of situations [24]. Each item is rated present or absent. Items rated present are then rated on a 1-9 scale, with higher scores indicating a greater degree of oppositional behavior. The RUPP-Autism Network adapted the HSQ for use in ASD populations (HSQ-ASD), expanding the measure to 24 items, yielding two 12-item subscales: Socially Inflexible and Demand Specific [25]. The HSQ-ASD has been used as a co-primary outcome measure in two randomized clinical trials and has demonstrated sensitivity to change with treatment [26, 27]. By convention, the HSQ-ASD is expressed as a per-item mean score (sum of the items rated 1-9 divided by 24). In this study, caregivers completed the HSQ-ASD at screening, baseline, and weeks 2,4 , and 8 .

Child and adolescent symptom inventory (CASI)-anxiety subscale The CASI $[28,29]$ is a reliable and valid, 132-item parent rating based on DSM-IV diagnostic criteria for a range of psychiatric disorders [30]. Items are scored: never (0) to very often (3); scores of 2 or 3 (occurring 'often' or 'very often') are considered clinically significant and contribute to a Symptom Count score for a specific disorder. The CASI contains 26 specific items related to 8 DSM-IV anxiety disorders. Using data from several multi-site trials $(N=415$ youth), Hallett and colleagues showed that 20 of these 26 items could be used as a reliable and valid index for anxiety in youth with ASD [31]. The measure demonstrated a normal distribution and good internal consistency in this clinical population. To our knowledge, the CASI has not been previously used as an outcome measure, yet insofar as it is comprised of DSM-IV anxiety symptoms, it is likely sensitive to change with treatment. In this study, caregivers completed the 20-item CASI-Anxiety subscale at baseline and endpoint (week 8 ).

Children's Yale-Brown obsessive-compulsive scales- modified for autism spectrum disorder (CYBOCS-ASD). The CY-BOCS-ASD is a clinician-rated, semi-structured measure developed to assess the severity of repetitive behavior in children with ASD. It was derived from the original CYBOCS designed to measure symptom severity in children with obsessive-compulsive disorder (OCD) [32]. Unlike the original version, the CYBOCS-ASD does not include assessment of obsessions. It retains the five severity items for repetitive behavior (time spent, interference with functioning, distress, resistance to performing repetitive behavior, and control over behavior). Each item is rated on a scale from 0 to 4 (total ranges from 0 to 20), with higher ratings indicating greater severity of repetitive behavior. The CY-BOCS-ASD scale has demonstrated reliability, validity, and sensitivity to change [33, 34]. The CY-BOCSASD was administered at baseline and endpoint by an independent evaluator masked to treatment allocation and to any discussion of adverse effects.

Children's sleep habits questionnaire (CSHQ)

The original CSHQ is a 33-item parent report measure of pediatric sleep disturbance that encompasses the medical and behavioral sleep disorders commonly encountered in school-aged children [35]. The CSHQ has been used in studies of sleep disturbance in children with ASD [36-38]. A recent psychometric evaluation of the CSHQ in a sample of 310 children with ASD ages 2-10 years identified 5 factors. Six items were dropped altogether. In our analysis, we used the total score of this modified 27-item scale [39]. Parents completed the CSHQ at screening and week 8 of the double-blind phase.

\section{Statistical analysis}

All randomly assigned subjects $(N=62)$ were included in the intent-to-treat analysis. Univariate exploratory data analysis was used to evaluate the distribution for the outcome variables of interest (HSQ-PDD, CASI-Anxiety, CYBOCS-ASD, and CSHQ ratings). $A$ repeated measures linear mixed model was used to determine the effects of treatment group and time (weeks 2, 4, and 8) on the HSQ per-item mean score. Least squares (LS) means from models were reported. Change from baseline across groups on the CYBOCS-ASD, CASI-Anxiety and CSHQ were evaluated using

\begin{tabular}{|c|c|c|c|c|c|}
\hline Guanfacine, $n=30$ Mean $(95 \% \mathrm{Cl})$ & $\%$ Change & Placebo, $n=32$ Mean $(95 \% \mathrm{Cl})$ & $\%$ Change & Group difference mean $(95 \% \mathrm{Cl})$ & $P$-value* \\
\hline \multicolumn{6}{|c|}{ HSQ-ASD ${ }^{a}$} \\
\hline $2.2(1.7-2.7)$ & $-35 \%$ & $3.0(2.5-3.5)$ & $-9 \%$ & $-0.8(-1.5$ to -0.1$)$ & 0.0316 \\
\hline $1.6(1.1-2.1)$ & $-53 \%$ & $2.5(1.9-3.0)$ & $-24 \%$ & $-0.8(-1.6$ to -0.1$)$ & 0.0209 \\
\hline $14.0(10.1-17.8)$ & & $15.8(11.8-19.8)$ & & & \\
\hline $10.8(8.7-12.9)$ & $-23 \%$ & $11.6(8.8-14.5)$ & $-27 \%$ & $-0.8(-4.3$ to 2.7$)$ & 0.64 \\
\hline \multicolumn{6}{|c|}{ CYBOCS-ASD ${ }^{c}$} \\
\hline $11.2(9.3-13.1)$ & & $10.9(9.0-12.9)$ & & & \\
\hline $8.5(6.9-10.1)$ & $-24 \%$ & $10.8(9.9-11.8)$ & $<-1 \%$ & $-2.3(-4.1$ to -0.5$)$ & 0.0143 \\
\hline $\begin{array}{l}\text { mean scores at baseline and least sq } \\
\text { score is per item mean from } 24 \text { item } \\
\text { ciety score is a total score of } 20 \text { items } \\
\text { ASD score is a total score of } 5 \text { items } \\
\text { ore is a total score for } 27 \text { items } \\
\text { LS means differences between guanfa } \\
\text { re bolded if statistically significant at p } \\
\text { the specified time points. }\end{array}$ & $\begin{array}{l}\text { uares (LS) me } \\
\text { s } \\
\text { aine and plac } \\
\text { less than or }\end{array}$ & $\begin{array}{l}\text { cebo groups } \\
\text { equal to } 0.05 \text {. The } p \text {-value is for dif }\end{array}$ & ferences betv & en adjusted LS means for the tw & eatment \\
\hline
\end{tabular}


Analysis of Covariance (ANCOVA), with baseline scores in the model. All model residuals were checked for normality. All analyses were conducted using SAS/STAT software, version 9.4. P-values of $\leq 0.05$ were accepted as statistically significant.

\section{RESULTS}

Eighty-one children were screened, and 62 eligible subjects (53 boys and 9 girls) were randomly assigned to GEXR $(N=30)$ or placebo $(N=32)$ (see supplemental Fig. 1 for CONSORT diagram). Subjects ranged in age from 5 to 14 years (mean $=8.5$ years [SD $=2.25]$ ). Baseline demographic and clinical characteristics did not significantly differ between treatment groups (see Table 1). The modal dose in both the guanfacine and placebo group at week 8 was $3 \mathrm{mg}$ daily. Drowsiness, fatigue, decreased appetite, irritability, and lowered diastolic blood pressure and heart rate were significantly more common in the GEXR group than the placebo group [10]. Four subjects in the placebo group discontinued early due to lack of efficacy, and 4 subjects in the GEXR discontinued early due to adverse effects $(n=2)$, lack of efficacy $(n=1)$, or study burden $(n=1)$. Two additional subjects in the GEXR group discontinued treatment due to adverse effects but remained in the study for assessment.

Between-group adjusted LS means for scores of oppositional behavior, anxiety, repetitive behavior, and sleep disturbance are summarized in Table 2. Parent ratings of oppositional behavior on the HSQ-ASD declined by $44 \%$ (3.4 to 1.9) in the GEXR group at week 8 (LS means difference, $p=0.004$ ) compared to $12 \%$ (from 3.3 to 2.9 ) in the placebo group (see Fig. 1). Repetitive behavior, as measured by the CYBOCS-ASD, showed a significantly greater decline in the GEXR group compared to placebo ( $24 \%$ vs. $<1 \%$, $p=0.0143$ ) (see Fig. 2). There was no group difference in change from baseline to Week 8 on the CASI-Anxiety $(p=0.64)$ or the CSHQ total score $(p=0.75)$.

\section{DISCUSSION}

Children with ASD frequently exhibit a range of emotional and behavioral problems, such as ADHD symptoms, oppositional behavior, anxiety, repetitive behaviors, and sleep disturbance.
We previously reported that GEXR is effective for reducing hyperactivity and impulsiveness in children with ASD [10]. The CGI-I defined positive response rates were comparable to methylphenidate in youth with ASD (approximately 50\%), but with a slightly lower frequency of discontinuation due to adverse effects (13.3 vs. $18 \%$ of subjects) [7]. In this study, we found that GEXR was superior to placebo in reducing oppositional behavior on the HSQ-ASD and repetitive behaviors on the CYBOCS-ASD in children with ASD.

As in ADHD, high levels of hyperactivity and impulsiveness can contribute to oppositional behavior in children with ASD. In a placebo-controlled trial of 461 children aged 6-17-year-old with $A D H D$, the use of GEXR as an adjunct to stimulant treatment resulted in significantly greater improvement in oppositional behavior, as measured by the oppositional subscale of the Conners' Parent Rating Scale-Revised: Long Form (CPRS-R:L), compared with stimulant treatment alone [14]. Compared to placebo, atomoxetine also showed significant improvement in oppositional behavior, as measured by the HSQ, in a sample of children with ASD and moderate or greater ADHD symptoms [5]. A prior controlled trial of methylphenidate in youth with ASD and ADHD symptoms did not find statistically significant improvement in ratings of oppositional behavior, though baseline scores on ratings of oppositional behavior were relatively low in that sample [40]. By contrast, in a study of extended-release methylphenidate in school-aged children with ASD, active medication was superior to placebo on parent and teacher ratings of oppositional behavior assessed with the Conners Parent Rating Scale- Revised and ADDH Comprehensive Teacher Rating Scale [8]. Collectively, these results suggest that GEXR may be an alternative to methylphenidate for monotherapy in children with ASD complicated by hyperactivity and oppositional behavior, particularly for those who do not show a positive response or cannot tolerate methylphenidate. It may also be useful in combined treatment with methylphenidate [14].

A large number of studies have evaluated and supported the use of atypical antipsychotics for the treatment of severe irritability in children with ASD. Most of the clinical trials of antipsychotics for irritability and aggressive behavior in ASD used the ABC-Irritability subscale and CGI-I as clinical endpoints, with

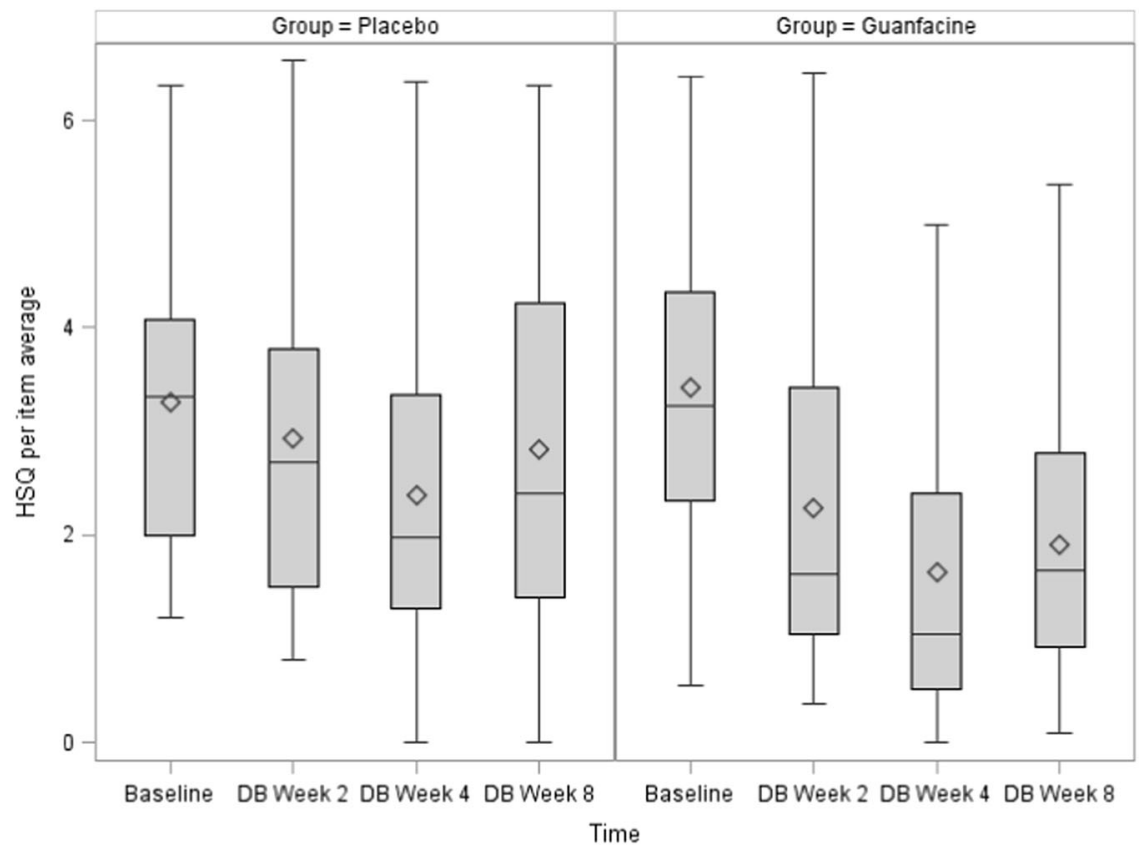

Fig. 1 Change in the HSQ-ASD per-item mean over time by treatment group $(P=0.0042)$ 


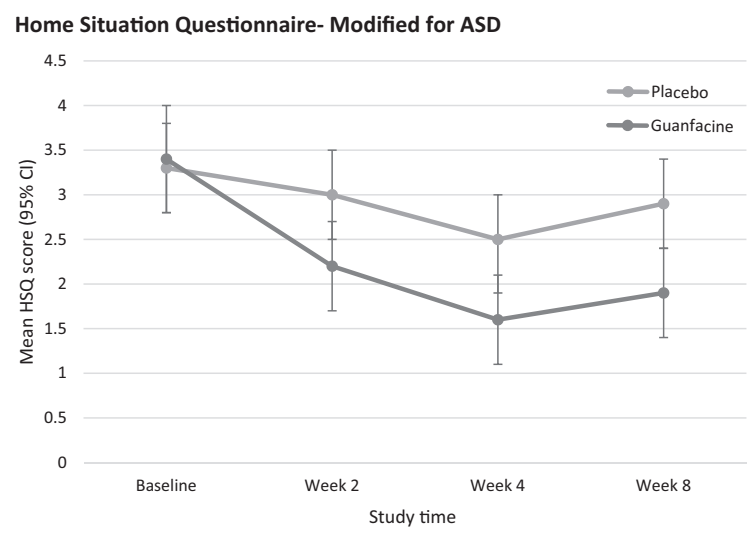

Note: Mean for baseline score and adjusted LS Means for follow-up scores plotted

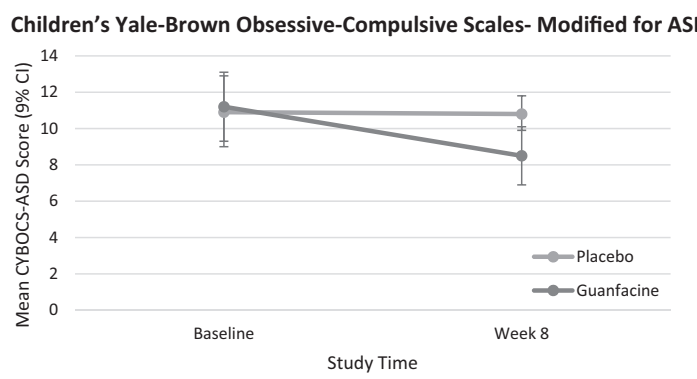

Note: Mean for baseline score and adjusted LS Means for follow-up score plotted

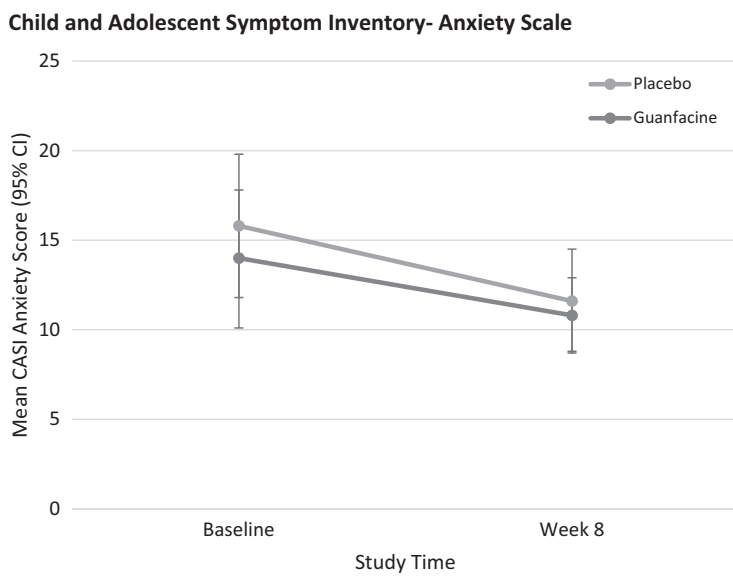

Note: Mean for baseline score and adjusted LS Means for follow-up score plotted

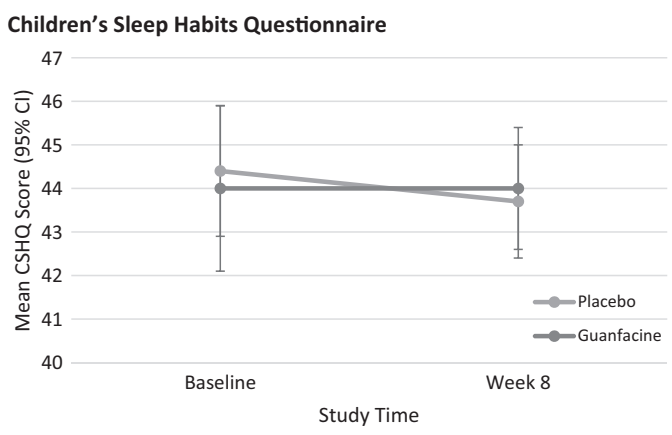

Note: Mean for baseline score and adjusted LS Means for follow-up score plotted

Fig. 2 Change in outcome measures over time. The mean for baseline score and adjusted LS means for follow-up score are plotted. The HSQ-ASD was assessed at baseline, week 4, week 8, and week 12. The CYBOCS-ASD, CASI-Anxiety, and CHSQ were assessed at baseline and week 8 aripiprazole having moderate effect sizes and risperidone having large effect sizes for these symptoms. In our report of primary findings for the GEXR study [10], we reported no statistically significant differences in irritability with treatment between the GEXR and placebo groups, as assessed by the ABC-irritability subscale $(\mathrm{ES}=0.27 ; p=0.20$ ). The HSQ assesses a qualitatively different set of behaviors, pertaining to non-compliance in everyday life (the term non-compliance is in the instructions on the measure) compared to the ABC-Irritability subscale which focuses on tantrums, mood lability, and to a lesser extent aggression and self-injury. In our psychometric evaluation of the HSQ-ASD $(N=242)$, we observed Pearson correlations of 0.46 and 0.42 for the $A B C$ Irritability and Hyperactivity subscales, respectively [25]. Taken together, GEXR does not appear as effective as antipsychotics for irritability as measured by the $A B C$ in youth with ASD, though may be beneficial for the whining or refusing often associated with oppositional behavior.

No statistically significant between-group differences were observed on the CASI-Anxiety scale, though parent-rated anxiety scores reflected relatively low levels of baseline anxiety in this sample. Thus, this study does not provide information on the effect of GEXR on children with more prominent anxiety symptoms. To improve the likelihood of detecting a clinically significant effect, future studies of guanfacine for the treatment of anxiety would benefit from the use of threshold scores for specific anxiety disorders as a study inclusion criterion.

Although the pre-treatment mean scores on the CYBOCS-ASD were relatively low, GEXR was modestly effective for reducing repetitive behaviors compared to placebo. In previous studies of methylphenidate, repetitive behavior did not improve despite improvement in measures of hyperactivity [7]. Here, it is possible that repetitive behavior improved independent of reduction in hyperactivity more generally, though this relationship was not specifically examined. The decline in repetitive behavior with GEXR was similar to the reduction reported in the RUPP risperidone trial [33]. However, concern for weight gain and related metabolic adverse effects limits the use of risperidone to children with serious behavioral problems [41].

In our previous report, we noted that drowsiness was a common adverse event in the GEXR group ( $N=26 / 30$ versus 3 / 32 for placebo). Parents also reported mid-sleep awakening in 9 of 32 subjects taking GEXR compared to $2 / 32$ for placebo [10]. In clinical practice, the alpha 2 receptor agonizts, clonidine and guanfacine, are commonly used off-label to help children fall asleep [42]. We included the CSHQ to assess the impact of GEXR on sleep. The results presented here do not support the use of GEXR as a sleep aid.

Given that the study was powered to detect an effect on the primary outcome measure, the $A B C$-Hyperactivity subscale, our ability to draw conclusions on the results from the analysis of these secondary outcomes is somewhat limited. In summary, GEXR appears effective for reducing oppositional behavior and repetitive behaviors, as well as hyperactivity in children with ASD accompanied by significant ADHD symptoms. Taken together, these results suggest that GEXR can be considered a first-line treatment for children with ASD who exhibit hyperactivity, as well as oppositional behavior or repetitive behavior.

\section{ACKNOWLEDGEMENTS}

The authors thank Courtney McCracken for her assistance with data analysis for this study.

\section{FUNDING}

The study was supported by NIMH grants to Dr. Scahill (R01MH083707), Dr. McDougle (RO1MH83739), Dr. McCracken (RO1MH083747), and Dr. King (R01MH86927); by a Yale Clinical and Transitional Science Award (UL1 RR024139) from the NIH National 
Center for Research Resources; and by Atlanta Clinical and Translational Science Institute, Emory University, which is supported by the NIH National Center for Advancing Translational Sciences under award UL1TR000454. Shire Pharmaceuticals provided active extended-release guanfacine and placebo. Dr. LCP receives research support from a Career Development Award with the North Carolina Translational and Clinical Sciences Institute, which is supported by NIH National Center for Advancing Translational Sciences under award UL1TR001111. Dr. LCP has received study drug from Nutramax, Inc. Dr. LS has served as a consultant for Bracket, Roche, and Shire, and he has served on the speaker's bureau for the Tourette Syndrome Association. Dr. JTM has served as a consultant for Dart Neuroscience and Roche; he has received research support from Roche; he has received study drug and matching placebo from Shire; and he has served on the speaker's bureau for the Tourette Syndrome Association. Dr. BK has received research support from Roche and Seaside Therapeutics.

\section{ADDITIONAL INFORMATION}

Supplementary information accompanies this paper at (https://doi.org/10.1038/ s41386-018-0039-3).

Conflict of interest: The authors declare that they have no conflict of interest.

Publisher's note: Springer Nature remains neutral with regard to jurisdictional claims in published maps and institutional affiliations.

\section{REFERENCES}

1. American Psychiatric Association. Diagnostic and statistical manual of mental disorders. 5th edn. Washington, DC: American Psychiatric Publishing; 2013.

2. Elsabbagh M, Divan G, Koh YJ, Kim YS, Kauchali S, Marcin C, et al. Global prevalence of autism and other pervasive developmental disorders. Autism Res. 2012;5:160-79.

3. Fombonne E. Epidemiology of pervasive developmental disorders. Pediatr Res. 2009;65:591-8.

4. Accordino RE, Kidd C, Politte LC, Henry CA, McDougle CJ. Psychopharmacological interventions in autism spectrum disorder. Expert Opin Pharmacother. 2016;17:937-52.

5. Handen BL, Aman MG, Arnold LE, Hyman SL, Tumuluru RV, Lecavalier L, et al. Atomoxetine, parent training, and their combination in children with autism spectrum disorder and attention-deficit/hyperactivity disorder. J Am Acad Child Adolesc Psychiatry. 2015;54:905-15.

6. Harfterkamp M, van de Loo-Neus G, Minderaa RB, van der Gaag RJ, Escobar R, Schacht $A$, et al. A randomized double-blind study of atomoxetine versus placebo for attention-deficit/hyperactivity disorder symptoms in children with autism spectrum disorder. J Am Acad Child Adolesc Psychiatry. 2012;51:733-41.

7. Research Units on Pediatric Psychopharmacology (RUPP) Autism Network Randomized, controlled, crossover trial of methylphenidate in pervasive developmental disorders with hyperactivity. Arch Gen Psychiatry. 2005;62: 1266-74.

8. Pearson DA, Santos CW, Aman MG, Arnold LE, Casat CD, Mansour R, et al. Effects of extended release methylphenidate treatment on ratings of attention-deficit/ hyperactivity disorder (ADHD) and associated behavior in children with autism spectrum disorders and ADHD symptoms. J Child Adolesc Psychopharmacol. 2013;23:337-51

9. Reichow B, Volkmar FR, Bloch $\mathrm{MH}$. Systematic review and meta-analysis of pharmacological treatment of the symptoms of attention-deficit/hyperactivity disorder in children with pervasive developmental disorders. J Autism Dev Disord. 2013;43:2435-41.

10. Scahill L, McCracken JT, King BH, Rockhill C, Shah B, Politte L, et al. Extendedrelease guanfacine for hyperactivity in children with autism spectrum disorder. Am J Psychiatry. 2015;172:1197-206.

11. Troost PW, Steenhuis MP, Tuynman-Qua HG, Kalverdijk LJ, Buitelaar JK, Minderaa $\mathrm{RB}$, et al. Atomoxetine for attention-deficit/hyperactivity disorder symptoms in children with pervasive developmental disorders: a pilot study. J Child Adolesc Psychopharmacol. 2006;16:611-9.

12. Martinez-Raga J, Knecht $C$, de Alvaro R. Profile of guanfacine extended release and its potential in the treatment of attention-deficit hyperactivity disorder. Neuropsychiatr Dis Treat. 2015;11:1359-70.

13. Wang $M$, Ramos BP, Paspalas $C D$, Shu $Y$, Simen A, Duque A, et al. Alpha2A-adrenoceptors strengthen working memory networks by inhibiting CAMP-HCN channel signaling in prefrontal cortex. Cell. 2007;129: 397-410.

14. Findling RL, McBurnett $K$, White C, Youcha S. Guanfacine extended release adjunctive to a psychostimulant in the treatment of comorbid oppositional symptoms in children and adolescents with attention-deficit/hyperactivity disorder. J Child Adolesc Psychopharmacol. 2014;24:245-52.

15. Strawn JR, Compton SN, Robertson B, Albano AM, Hamdani M, Rynn MA. Extended release guanfacine in pediatric anxiety disorders:a pilot, randomized, placebo-controlled trial. J Child Adolesc Psychopharmacol. 2017;27:29-37.

16. Connor DF, Grasso DJ, Slivinsky MD, Pearson GS, Banga A. An open-label study of guanfacine extended release for traumatic stress related symptoms in children and adolescents. J Child Adolesc Psychopharmacol. 2013;23:244-51.

17. Murphy TK, Fernandez TV, Coffey BJ, Rahman O, Gavaletz A, Hanks CE, Tillberg CS, Gomez LI, Sukhodolsky DG, Katsovich L, Scahill L. Extended-release guanfacine does not show a large effect on tic severity in children with chronic tic disorders. J Child Adolesc Psychopharmacol. 2017;27:762-70. https://doi.org/10.1089/ cap.2017.0024.

18. Efron D, Lycett $K$, Sciberras $E$. Use of sleep medication in children with ADHD. Sleep Med. 2014;15:472-5.

19. Rugino TA. Effect on primary sleep disorders when children with ADHD are administered guanfacine extended release. J Atten Disord. 2014;22:14-24. https://doi.org/10.1177/1087054714554932.

20. Aman MG, Singh NN, Stewart AW, Field CJ. The aberrant behavior checklist: a behavior rating scale for the assessment of treatment effects. Am J Ment Defic. 1985;89:485-91.

21. Lord C, Rutter M, Goode S, Heemsbergen J, Jordan H, Mawhood L, et al. Autism diagnostic observation schedule: a standardized observation of communicative and social behavior. J Autism Dev Disord. 1989;19:185-212.

22. Rutter MBA, Lord C. The social communication questionnaire. Los Angeles, CA: Western Psychological Services; 2003.

23. Guy W. Clinical Global Impressions (CGI) Scale. In:Rush J, editors. Psychiatric measures. Washington, D.C: APA; 2000.

24. Barkley RA, Edelbrock C. Assessing situational variation in children's problem behaviors: the Home and School Situations Questionnaires. In: Prinz R, editors. Advances in behavioral assessment of children and families. Greenwich, CT: JAI Press Inc.; 1987. pp. 157-76.

25. Chowdhury M, Aman MG, Lecavalier L, Smith T, Johnson C, Swiezy N, et al. Factor structure and psychometric properties of the revised Home Situations Questionnaire for autism spectrum disorder: the home situations questionnaire-autism spectrum disorder. Autism. 2016;20:528-37.

26. Aman MG, McDougle CJ, Scahill L, Handen B, Arnold LE, Johnson C, et al. Medication and parent training in children with pervasive developmental disorders and serious behavior problems: results from a randomized clinical trial. J Am Acad Child Adolesc Psychiatry. 2009;48:1143-54.

27. Bearss K, Johnson C, Smith T, Lecavalier L, Swiezy N, Aman M, et al. Effect of parent training vs parent education on behavioral problems in children with autism spectrum disorder: a randomized clinical trial. JAMA 2015;313:1524-33.

28. Gadow KDSJ. Child symptom inventory-4. Stony Brook, NY: Checkmate Plus; 1994.

29. Gadow KDSJ. Adolescent Symptom Inventory-4 screening manual. Stony Brook, NY: Checkmate Plus; 1997.

30. Sprafkin J, Gadow KD, Salisbury H, Schneider J, Loney J. Further evidence of reliability and validity of the Child Symptom Inventory-4: parent checklist in clinically referred boys. J Clin Child Adolesc Psychol. 2002;31:513-24.

31. Hallett V, Lecavalier L, Sukhodolsky DG, Cipriano N, Aman MG, McCracken JT, et al. Exploring the manifestations of anxiety in children with autism spectrum disorders. J Autism Dev Disord. 2013;43:2341-52.

32. Scahill L, Riddle MA, McSwiggin-Hardin M, Ort SI, King RA, Goodman WK, et al. Children's Yale-Brown obsessive compulsive scale: reliability and validity. J Am Acad Child Adolesc Psychiatry. 1997;36:844-52.

33. McDougle CJ, Scahill L, Aman MG, McCracken JT, Tierney E, Davies M, et al. Risperidone for the core symptom domains of autism: results from the study by the autism network of the research units on pediatric psychopharmacology. Am J Psychiatry. 2005;162:1142-8.

34. Scahill L, McDougle CJ, Williams SK, Dimitropoulos A, Aman MG, McCracken JT, et al. Children's Yale-Brown obsessive compulsive Scale modified for pervasive developmental disorders. J Am Acad Child Adolesc Psychiatry. 2006;45: 1114-23.

35. Owens JA, Spirito A, McGuinn M. The children's sleep habits questionnaire (CSHQ): psychometric properties of a survey instrument for school-aged children. Sleep. 2000;23:1043-51.

36. Giannotti F, Cortesi F, Cerquiglini A, Vagnoni C, Valente D. Sleep in children with autism with and without autistic regression. J Sleep Res. 2011;20:338-47.

37. Malow BA, Marzec ML, McGrew SG, Wang L, Henderson LM, Stone WL. Characterizing sleep in children with autism spectrum disorders: a multidimensional approach. Sleep. 2006;29:1563-71.

38. Reed HE, McGrew SG, Artibee K, Surdkya K, Goldman SE, Frank K, et al. Parentbased sleep education workshops in autism. J Child Neurol. 2009;24:936-45. 
39. Johnson CR, DeMand A, Lecavalier L, Smith T, Aman M, Foldes E, et al. Psychometric properties of the children's sleep habits questionnaire in children with autism spectrum disorder. Sleep Med. 2016;20:5-11.

40. Posey DJ, Aman MG, McCracken JT, Scahill L, Tierney E, Arnold LE, et al. Positive effects of methylphenidate on inattention and hyperactivity in pervasive developmental disorders: an analysis of secondary measures. Biol Psychiatry. 2007;61:538-44.
41. Scahill L, Jeon S, Boorin SJ, McDougle CJ, Aman MG, Dziura J, et al. Weight gain and metabolic consequences of risperidone in young children with autism spectrum disorder. J Am Acad Child Adolesc Psychiatry. 2016;55: 415-23.

42. Fiks AG, Mayne SL, Song L, Steffes J, Liu W, McCarn B, et al. Changing patterns of alpha agonist medication use in children and adolescents 2009-2011. J Child Adolesc Psychopharmacol. 2015;25:362-7. 